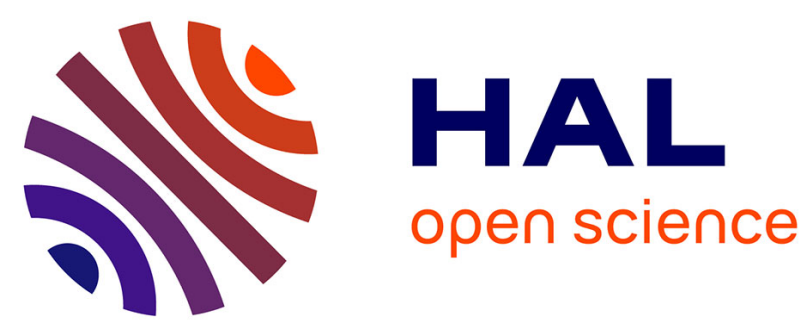

\title{
mTOR inhibitors may benefit kidney transplant recipients with mitochondrial diseases
}

\author{
Simon C. Johnson, Frank Martinez, Alessandro Bitto, Brenda Gonzalez, \\ Cagdas Tazaerslan, Camille Cohen, Laure Delaval, José Timsit, Bertrand \\ Knebelmann, Fabiola Terzi, et al.
}

\section{To cite this version:}

Simon C. Johnson, Frank Martinez, Alessandro Bitto, Brenda Gonzalez, Cagdas Tazaerslan, et al.. mTOR inhibitors may benefit kidney transplant recipients with mitochondrial diseases. Kidney International, 2019, 95, pp.455 - 466. 10.1016/j.kint.2018.08.038 . hal-03486913

\section{HAL Id: hal-03486913 https://hal.science/hal-03486913}

Submitted on 20 Dec 2021

HAL is a multi-disciplinary open access archive for the deposit and dissemination of scientific research documents, whether they are published or not. The documents may come from teaching and research institutions in France or abroad, or from public or private research centers.
L'archive ouverte pluridisciplinaire HAL, est destinée au dépôt et à la diffusion de documents scientifiques de niveau recherche, publiés ou non, émanant des établissements d'enseignement et de recherche français ou étrangers, des laboratoires publics ou privés.

\section{(ㅇ)(1) $\$$}

Distributed under a Creative Commons Attribution - NonCommercial| 4.0 International 
[QUERY TO AUTHOR: title and abstract rewritten by Editorial Office - not subject to change] mTOR inhibitors may benefit kidney transplant recipients with mitochondrial diseases

Simon C. Johnson $\mathrm{PhD}^{1,9}$, Frank Martinez $\mathrm{MD}^{2 *}$, Alessandro Bitto $\mathrm{PhD}^{3 *}$, Brenda Gonzalez $\mathrm{PhD}^{1 *}$, Cagdas Tazaerslan $\mathrm{PhD}^{1}$, Camille Cohen $\mathrm{MD}^{2}$, Laure Delaval $\mathrm{MD}^{2}$, José Timsit MD, $\mathrm{PhD}^{4,5}$, Bertrand Knebelmann $\mathrm{MD}, \mathrm{PhD}^{2,5,6}$, Fabiola Terzi $\mathrm{MD}, \mathrm{PhD}^{6}$, Tarika Mahal $\mathrm{PhD}^{1}$, Yizhou Zhu $\mathrm{PhD}^{1}$, Philip G. Morgan MD, $\mathrm{PhD}^{9,10}$, Margaret M. Sedensky MD, $\mathrm{PhD}^{9,10}$, Matt Kaeberlein $\mathrm{PhD}^{3}$, Christophe Legendre $\mathrm{MD}^{2,5,6}$, Yousin Suh $\mathrm{PhD}^{1,7,8}$, Guillaume Canaud MD, $\mathrm{PhD}^{2,5,6} \uparrow$

${ }^{1}$ Department of Genetics, Albert Einstein College of Medicine, Bronx, NY, US

${ }^{2}$ Service de Néphrologie Transplantation Adultes, Hôpital Necker-Enfants Malades, Paris, France

${ }^{3}$ Department of Pathology, University of Washington, Seattle, WA, US

${ }^{4}$ Service d'Immunologie-Diabétologie, Hôpital Cochin, Paris, France

${ }^{5}$ Université Paris Descartes, Sorbonne Paris Cité, Hôpital Necker-Enfants Malades, Paris, France

${ }^{6}$ INSERM U1151, Institut Necker Enfants Malades, Hôpital Necker-Enfants Malades, Paris, France

${ }^{7}$ Department of Ophthalmology \& Visual Sciences, Albert Einstein College of Medicine, Bronx, NY, US

${ }^{8}$ Department of Medicine, Endocrinology, Albert Einstein College of Medicine, Bronx, NY, US

${ }^{9}$ Center for Integrative Brain Research, Seattle Children's Research Institute, Seattle, WA

${ }^{10}$ Department of Anesthesiology and Pain Medicine, University of Washington, Seattle, WA

* These authors contributed equally to this work.

$\dagger$ Corresponding author:

Dr. Guillaume Canaud

Institut National de la Santé et de la Recherche Médicale U1151

Service de Néphrologie Transplantation Adultes, Hôpital Necker-Enfants Malades

Université Paris Descartes

149 rue de Sèvres, 75015 Paris, France.

Email: guillaume.canaud@inserm.fr

Word count: 3055 


\begin{abstract}
Mitochondrial diseases represent a significant clinical challenge. Substantial efforts have been devoted to identifying therapeutic strategies for mitochondrial disorders, but effective interventions have remained elusive. Recently, we reported attenuation of disease in a mouse model of the human mitochondrial disease Leigh syndrome through pharmacological inhibition of the mechanistic target of rapamycin (mTOR). The human mitochondrial disorder MELAS/MIDD (Mitochondrial Encephalopathy with Lactic Acidosis and Stroke-like Episodes/ Maternally Inherited Diabetes and Deafness) shares many phenotypic characteristics with Leigh syndrome. MELAS/MIDD often leads to organ failure and transplantation and there are currently no effective treatments. To examine the therapeutic potential of mTOR inhibition in human mitochondrial disease, four kidney transplant recipients with MELAS/MIDD were switched from calcineurin inhibitors to mTOR inhibitors for immunosuppression. Primary fibroblast lines were generated from patient dermal biopsies and the impact of rapamycin was studied using cellbased endpoints. Metabolomic profiles of the four patients were obtained before and after the switch. pS6, a measure of mTOR signaling, was significantly increased in MELAS/MIDD cells compared to controls in the absence of treatment, demonstrating mTOR overactivation. Rapamycin rescued multiple deficits in cultured cells including mitochondrial morphology, mitochondrial membrane potential, and replicative capacity. Clinical measures of health and mitochondrial disease progression were improved in all four patients following the switch to an mTOR inhibitor. Metabolomic analysis was consistent with mitochondrial function improvement in all patients.
\end{abstract}

Key Words: mitochondria, chronic kidney disease 


\section{Significance Statement}

Mitochondrial diseases represent a challenge in human medicine. Recent studies have indicated that the mechanistic target of rapamycin (mTOR) pathway is upregulated in a number of mitochondrial diseases, and its inhibition is an effective therapeutic strategy in animal models. Mitochondrial diseases are clinically and genetically heterogeneous that can lead to organ failure and transplantation. mTOR inhibitors are used in humans as anti-transplant rejection drugs. We show that fibroblasts derived from patients with mitochondrial diseases exhibit hyperactive mTOR and cellular and mitochondrial defects, attenuated by mTOR inhibition. We further find that patients switched to mTOR inhibitors for transplant management show clinical improvement. This study provides the first direct evidence that mTOR inhibition is beneficial in human mitochondrial disease patients. 


\section{Introduction}

Mitochondrial diseases are a clinically and genetically heterogeneous group of disorders that arise as a result of mitochondrial dysfunction ${ }^{1}$. Inherited mitochondrial diseases can be caused by mutations of mitochondrial DNA (mtDNA) or of nuclear genes that encode mitochondrial proteins, with an overall prevalence estimated at approximately 1 in 5,000 ${ }^{1}$. The clinical phenotypes of patients affected by mitochondrial disorders are considerably heterogeneous ${ }^{2}$. Individuals with mitochondrial disorders resulting from mutation of mtDNA may harbor a mixture of mutated and wild-type mtDNA within each cell. The percentage of mutant mtDNA varies between individuals, as well as among organs and tissues within the same individual, contributing to the varied clinical phenotype ${ }^{3}$.

Mitochondrial encephalomyopathy with lactic acidosis and stroke-like episodes (MELAS) syndrome is a genetic disorder that results from mutations affecting mtDNA encoding NADH dehydrogenase or transfer RNAs ${ }^{4}$. The most frequent mutation in patients with MELAS syndrome is a $3243 \mathrm{~A}>\mathrm{G}$ mtDNA point mutation, but over a dozen distinct causal genetic variants have been linked to the disease. Patients with MELAS syndrome typically undergo normal developmental but develop bilateral deafness in late childhood, diabetes in early adulthood, progressive neurological disabilities from the third decade of life with migraines, seizures, stroke-like episodes and encephalopathy, heart failure, and progressive kidney failure ${ }^{4}$. Not all MELAS patients will follow this clinical pattern with some showing a limited phenotype, such as maternally inherited diabetes and deafness (MIDD) ${ }^{5}$.

Currently, there is no effective treatment for mitochondrial diseases of any etiology and management is mainly supportive. In small uncontrolled studies, vitamins $\mathrm{C}$ and $\mathrm{K}$, thiamine, riboflavin, and ubiquinone have shown varying degrees of benefit in individual cases ${ }^{6}$. 
Recent experimental studies have demonstrated that reduced mTOR signaling through caloric restriction or genetic manipulation rescues the lifespan of yeast harboring mutations in genes encoding mitochondrial proteins ${ }^{7}$. Furthermore, we recently reported a robust attenuation of disease in a mouse model of the human mitochondrial disease Leigh syndrome ( $N d u f s 4^{-/-}$mice) using pharmacological inhibition of $\mathrm{mTOR}^{8,9}$. We observed that the $N d u f_{s} 4^{-/}$mice show increased tissue mTOR activation in affected tissue (brain) associated with metabolic defects and progressive neurological disease. Importantly, we found that rapamycin, a specific inhibitor of mTOR, substantially delayed the onset of neurological symptoms and lesions and extended the lifespan of the $N d u f_{s} 4^{-1-}$ mice ${ }^{8}$. These data where then confirmed in iPS cells from patients with Leigh syndrome ${ }^{10}$.

Rapamycin is an immunosuppressive drug used after solid organ transplantation to prevent allograft rejection ${ }^{11,12}$. Thus, transplant recipients receiving rapamycin or derivatives present a unique opportunity to study the effects of mTOR inhibition on human mitochondrial diseases. In this study, we identified four kidney transplant recipients with MELAS/MIDD syndrome. The four patients had stable allografts with an immunosuppressive regimen based on calcineurin inhibitors but their global health status was declining rapidly. Based on the findings in the mouse model we examined activation of the mTOR pathway in patient derived fibroblasts and switched the immunosuppression regimen from calcineurin inhibitors to mTOR inhibitors accordingly. 


\section{Results}

\section{Patients}

Patient 1 was a man born in 1962 and was diagnosed with a MELAS syndrome in 1996 (m.3243A $>\mathrm{G}$ mutation) (Table 1). Heteroplasmy was determined in three different samples: blood leukocytes (10-15\% mutant mtDNA), urine epithelial cells (5-10\% mutant mtDNA) and cheek cells (40-45\% mutant mtDNA). At the time of diagnosis, the patient had severe hearing impairment, diabetes years and kidney dysfunction with proteinuria. The kidney biopsy performed in 1996 showed focal and segmental glomerulosclerosis (FSGS). The patient had a first stroke-like episode in 2001. He reached end stage renal disease in 2004 and began hemodialysis. In 2005, he received a kidney from a deceased donor. The patient had no evidence for cardiac dysfunction but had muscle weakness. His immunosuppressive regimen was based on steroids, mycofenolate mofetil, and tacrolimus. Karnofsky and ECOG scores were $90 \%$ and 1, respectively, at the time of transplant. Following transplantation, the patient did not experience any complications related to immunosuppression and the estimated glomerular filtration rate (eGFR) remained stable at approximately $72 \mathrm{ml} / \mathrm{min}$ during the subsequent years. However, his general condition was found to be declining dramatically with considerable weight loss $(-18 \mathrm{~kg}$, $\Delta:-30 \%$ ), anorexia, and excessive fatigability. Biological markers, such as serum albumin and prealbumin, showed severe malnutrition. We excluded cancer, chronic infection, inflammation, and depression as a cause of denutrition. The patient's Karnofsky and ECOG scores dropped to $30 \%$ and 3, respectively. We concluded that the cachexia was related to progression of MELAS syndrome.

Patient 2 was the sister of patient 1 . She was born in 1954 and MELAS syndrome was diagnosed at the same time of her brother's diagnosis in 1996 (m.3243A $>\mathrm{G}$ mutation) (Table 1). 
Heteroplasmy was determined in three different samples: blood leukocytes $(<5 \%$ mutant mtDNA), urine epithelial cells (15-20\% mutant mtDNA) and cheek cells (25-30\% mutant mtDNA). She had hearing loss, diabetes, and severe renal impairment with proteinuria. Kidney biopsy revealed FSGS with severe interstitial fibrosis. She had a first stroke like episode in 1999. She reached end stage renal disease in 2001 and received a first kidney transplantation in 2003. In 2010, she had a second stroke like episode. The patient had no evidence for heart dysfunction but had muscle weakness. She reached end stage renal failure and received a kidney transplantation. Her immunosuppressive regimen included steroids, azathioprine and cyclosporine. The Karnofsky and ECOG scores at the time of transplantation were $90 \%$ and 1 respectively. Following transplantation, she did not experience any complication related to immunosuppression and the eGFR remained stable at approximately $78 \mathrm{ml} / \mathrm{min}$ for several years. However, such as for her brother, we observed a progressive anorexia with severe body weight loss $(-30 \mathrm{~kg}, \Delta:-50 \%)$, a dramatic decrease in nutrition markers, and increase in fatigability. We excluded cancer, infection or depression as a cause of cachexia. The Karnofsky score and ECOG scores dropped to $30 \%$ and 3 respectively. We therefore concluded that the phenotype was related to MELAS syndrome progression.

Patient 3 was a man born in 1965 who was diagnosed with a mitochondrial disorder in 1998 (m.3243A $>$ G mutation) (Table 1). Heteroplasmy was determined in two different tissues: blood leukocytes (15-20\% mutant mtDNA) and cheek cells (30-35\% mutant mtDNA). At the time of diagnosis, the patient had severe hearing impairment, chronic kidney disease with proteinuria and diabetes. A kidney biopsy performed in 2001 showed FSGS lesion. He underwent hemodialysis in 2008 and received a first kidney transplantation from a deceased donor 2 years later. Progressively, he developed severe and refractory systolic heart dysfunction related to 
mitochondrial disease. He has never had stroke like episode and presented muscle weakness. Given the lack of stroke-like episodes he was classified as having a MIDD syndrome according to criteria ${ }^{5}$. He underwent hemodialysis and received a kidney transplantation from a deceased donor. The Karnofsky and ECOG scores at the time of transplantation were $90 \%$ and 1 respectively. The immunosuppressive regimen included steroids, mycofenolate mofetil, and tacrolimus. Following the transplantation, the patient did not experience any complication related to immunosuppression and the eGFR remained stable at approximately $56 \mathrm{ml} / \mathrm{min}$. However, systolic heart function continued to worsen leading to severe congestive heart failure. His systolic ejection fraction dropped progressively from 42 to $30 \%$ and was resistant to standard treatment. In addition to weight gain related to edema secondary to heart failure, the general condition of the patient was dramatically worsening with excessive fatigability and severe markers of malnutrition. His Karnofsky score and ECOG scores dropped to $40 \%$ and 2 respectively. Following multiple exams, we concluded that his symptoms were related to progression of MELAS syndrome.

Patient 4 was a man born in 1955 who was diagnosed with a MELAS in 1999 (m.3243A>G mutation) (Table 1). Heteroplasmy was determined in two different samples, blood leukocytes (10-15\%, mutant mtDNA) and cheek cells (40-45\% mutant mtDNA). At the time of diagnosis the patient presented with hearing impairment, kidney dysfunction with proteinuria and diabetes. A kidney biopsy was performed in 2002 and showed FSGS lesion. He had a stroke like episode in 2008. He underwent hemodialysis in 2014 and received a first kidney transplantation from a deceased donor 1 year later. The immunosuppressive regimen included steroids, mycofenolate mofetil, and tacrolimus. The Karnofsky and ECOG scores at the time of transplantation were 90\% and 1 respectively. 
Cellular effects of blocking mTOR overactivation in primary cultured fibroblasts with m.3243A $>$ G mutation

Hyperactive mTOR signaling in primary fibroblasts from patients with m.3243A>G mutation

In our previous work we found mTOR to be hyperactivated in whole brain lysates of the Leigh syndrome mouse model ${ }^{8}$. To examine if the mTOR pathway was also hyperactive in primary MELAS/MIDD cells we cultured primary fibroblasts from patients and control individuals and collected protein lysates for western blotting. Importantly, we observed that the MELAS/MIDD cells show a significant increase in phosphorylation of ribosomal protein S6 (rpS6) compared to control fibroblasts, indicative of hyperactivated mTOR signaling (Fig. 1A; dose-response in Supplementary Fig. 1 and additional targets in Supplementary Fig. 2;).

MELAS/MIDD and controls cells were then treated for 48 hours with DMSO, rapamycin, or cyclosporin A. Cyclosporin A was included as a control for the immunosuppressive therapy patients were receiving prior to the start of this study. Cyclosporin A had no significant impact on pS6/S6, while 48 hours of rapamycin treatment reduced pS6 levels in all cell lines, as anticipated.

Rescue of mitochondrial morphology and membrane potential by short-term rapamycin treatment with no impact on heteroplasmy levels

To examine the impact of mTOR inhibition on mitochondrial morphology and membrane potential, a general measure of mitochondrial function, we treated dermal fibroblasts with rapamycin or DMSO. Cells were stained with TMRE, a marker of mitochondrial membrane potential, and 10-NAO, a marker of the inner mitochondrial membrane which acts as a membrane potential insensitive marker of mitochondrial mass (Fig. 1B and 1C). MELAS/MIDD lines show reduced membrane potential, determined by TMRE staining intensity, and abnormal 
morphology, characterized by swelling, fragmentation and the presence of depolarized (low TMRE staining) mitochondria (Supplementary Fig. 3). Treatment with rapamycin attenuated these phenotypes in all MELAS/MIDD fibroblasts while having no overt impact on the control cells (Fig. 1B and 1C). Electron microscopy also indicated that rapamycin treatment is associated with a partial rescue of mitochondria morphology and cytoskeletal fibers organization in fibroblasts from MELAS/MIDD patients (Supplementary Fig. 4).

mTOR inhibition is associated with induction of autophagy and mitophagy. Previously, we demonstrated that rescue of mitochondrial disease in a mouse model was independent of any direct changes to primary mitochondrial defects ${ }^{8}$, arguing against the model that selective removal of defective mitochondria underlies the benefits of mTOR inhibition. Consistent with this model, the rescue of membrane potential was not associated with a change in levels of m.3243A $>$ G heteroplasmy (Fig. 1D-F).

Hyperactive mTOR signaling in primary fibroblasts from pediatric mitochondrial disease patients

To determine whether hyperactive mTOR is a shared feature in mitochondrial disease fibroblast lines we obtained three control primary fibroblast lines and five pediatric mitochondrial disease primary fibroblast lines from the Coriell Institute Cell Repository. This set includes three Leigh syndrome lines, one ataxia and cerebellar hypoplasia Complex I line, and a Kearn-Sayre syndrome line (Table 2). Interstingly, we found that pediatric mitochondrial disease cells also demonstrated a significant increase in pS6 compared to control fibroblasts. These results indicate that hyperactivated mTOR signaling is a shared feature of genetically distinct mitochondrial disorders (Fig. 1G).

Attenuation of early replicative senescence in MELAS/MIDD fibroblast 
To determine whether long-term rapamycin treatment could rescue additional cellular phenotypes in MELAS/MIDD cells we performed replicative senescence assays to measure cellular replicative capacity. Primary MELAS/MIDD fibroblast lines lose the ability to grow at early population doublings compared to control fibroblasts, indicating a dramatic reduction in proliferative capacity and premature senescence compared to control lines (Fig. 2A). Rapamycin increased proliferative capacity in both control and MELAS/MIDD lines, but, importantly, the relative increase in MELAS/MIDD cells was substantially greater than in controls (Fig. 2A-B). Interestingly, cyclosporin A slightly increased proliferative capacity in all cell lines, but to a similar level in control and MELAS/MIDD cells.

A recent study investigating senescence in the setting of non-physiological models which induce mitochondrial dysfunction in cultured cells found that presence or absence of pyruvate governs a cellular decision to proliferate or senesce ${ }^{13}$. Using media supplemented with pyruvate we found that neither the shortened lifespan of MELAS/MIDD fibroblasts nor the lifespan increase resulting from rapamycin treatment were impacted, suggesting key differences between senescence in genuine mitochondrial disease cells versus models of mitochondrial stress (Supplementary Fig. 5).

In addition to measuring replicative capacity, we examined the effects of rapamycin on markers of senescence - senescence associated beta-galactosidase (SA $\beta$-Gal) staining, accumulation of actin-cytoskeleton stress fibers (detected by phalloidin staining), nuclear morphology defects

(nuclear 'blebbing'), expression of $p 16^{I N K 4 a}$ and expression of the senescence associated factor p21/Cip1. In each case the MELAS/MIDD lines show features consistent with premature cellular senescence that were attenuated by rapamycin treatment (Fig. 2C-G and Supplementary Fig. 6). 


\section{Attenuation of early replicative senescence in MELAS/MIDD fibroblast}

To determine whether the observed benefits to replicative capacity result from changes in m.3243A $>$ G heteroplasmy levels we collected DNA samples at each passage (every other population doubling, PD) across the duration of the replicative lifespan study. At the completion of the study we assessed the mutant mitochondrial DNA load as a function of PD in MD1, the line with the highest levels of mutant DNA at the start of the study (Fig. 2H). Suprisingly, m.3243A $>\mathrm{G}$ levels did not change throughout the duration of the study in either DMSO or rapamycin treated cells (Fig. 2I), demonstrating that our culture conditions did select against mutant DNA and indicating that the beneficial effects of rapamycin treatment are not a result of altered heteroplasmy levels.

\section{Clinical impact of mTOR inhibition in transplant recipients with MELAS/MIDD}

Based on the in vitro studies we decided to stop calcineurin inhibitors in all patients and to introduce either rapamycin or everolimus. Only a few weeks after introducing mTOR inhibitors we observed a dramatic improvement in the general condition of the four patients. Patients 1 and 2 started to quickly gain weight, without edema, and recover their appetites. Anorexia progressively disappeared as well as the general asthenia. Clinical tests showed a marked improvement in the serum levels of prealbumin and albumin. The Karnofsky and ECOG scores of patients 1 and 2 increased from 30 to $60 \%$ and from 3 to 1 within 20 months (Fig. 3, Table 1). Improvement was also visible in patient 3 with an enhancement of the general condition (Fig. 3, Table 1). The patient lost weight due to the reduction of the edema related to a progressive amelioration of the heart systolic ejection fraction from 30 to $48 \%$. No additional treatments for congestive heart failure were added during the period, supporting a beneficial effect of mTOR inhibitors in this context. The Karnofsky and ECOG scores of patient 3 increased from 40 to 
$80 \%$ and from 2 to 1 within the 18 months. Patient 4 was switched only few months after transplantation compared to others but we could observe an improvement of the global status compared to the dialysis period. However, in this specific case it is difficult to separate the amelioration linked to the transplantation or the introduction of mTOR inhibitors or both (Fig. 3, Table 1). Importantly, in all patients the eGFR remainded stable after the switch meaning that the clinical improvement was not related to a gain of kidney function (Table 1).

\section{Metabolomic impact of mTOR inhibition in transplant recipients with MELAS}

Metabolic profiling of patients' sera also provided additional evidence of improved clinical outcome upon switching to sirolimus/everolimus (Fig. 3G and Supplementary Fig. 7). Serum 3-nitro-tyrosine, a marker of oxidative stress, was significantly reduced by mTOR inhibition (Fig. 3G and Supplementary Fig. 7), consistent with our in vitro results on mitochondrial membrane potential and morphology. Several markers of amino acid metabolism were also significantly altered by rapamycin treatment (Fig. 3G and Supplementary Fig. 7). In particular, levels of 1- and 3-methyl-histidine were significantly reduced upon switching to rapamycin, consistent with decreased muscle protein breakdown (Fig. 3G and Supplementary Fig. 7). Similar to our previous findings in $N d u f s 4^{-/}$mice $^{8,9}$, free fatty acid levels were generally elevated by rapamycin treatment, though not significantly, while TCA intermediates were reduced (Fig. 3G and Supplementary Fig. 7). Lastly, rapamycin treatment increased the levels of circulating glucose in all 4 patients (Fig. 3G and Supplementary Fig. 7), also consistent with our prior findings in the $N d u f s 4^{-/-}$model $^{8,9}$. Notably, a robust reduction in glucose consumption was also observed in the fibroblasts treated with rapamycin, indicating that the altered glucose handling resulting from mTOR inhibition is at least partly cell autonomous (Supplementary Fig. 8). 


\section{Discussion}

This study provides the first direct evidence supporting mTOR inhibition as a promising therapeutic strategy in human mitochondrial disease. While this work will need to be followed by studies involving larger sample sizes, and MELAS/MIDD patients who have not undergone organ transplantation, our results indicate that mTOR inhibition may be a safe and effective means to improve health and quality of life in transplant patients with MELAS/MIDD.

In addition, our cell-based studies provide new mechanistic insight into the cellular impact of MELAS/MIDD and the mechanism of benefit by rapamycin. We found $\mathrm{S} 6$ to be hyperphosphorylated in MELAS/MIDD fibroblasts and report premature cellular senescence to be one cellular pathological feature of MELAS/MIDD disease. We also observed MELAS/MIDD cells to have overtly defective mitochondrial membrane potential and morphology which was rescued by short-term mTOR inhibition. We were able to show that the improvement with rapamycin was not associated with a reduction in relative levels of mutant mitochondrial DNA, arguing against selective mitophagy of dysfunctional mitochondria as a mechanistic explanation.

Defining the precise mechanism that mediates the benefits of mTOR inhibition in mitochondrial disease is an active area of research that may lead to more targeted intervention strategies. We foused here on fibroblasts but we cannont formally exclude a positive impact of mTOR inhibitors on the immune systeme in this population.

The unique unbiased metabolomics profile of the patients before and after the switch to mTOR inhibitors provide additional evidences of mitochondrial improvement. We observed a drop in the levels of 1 and 3-methyl-histidine, a marker of muscle protein breakdown, suggesting that mTOR inhibition in this context prevent muscle wasting in MELAS/MIDD patients. We also 
found a decrease in oxidative stress markers arguing for mitochondrial respiratory chain improvement. Importantly, the results were consistent with those observed in the $\mathrm{Ndufs} 4^{--}$mice before and after rapamycin treatment ${ }^{8}$.

Finally, our study suggest that pre-screening of mTOR hyperactivation in patient samples and response to mTOR inhibitors may be a viable approach to personalized medicine in mitochondrial disease patients. More basic research is needed to determine which forms of mitochondrial disease will be refractory to mTOR inhibition, but it seems likely that those associated with hyperactive mTOR are the most likely to benefit. 


\section{Methods}

\section{Patients and Data Collection}

Our institutional review board approved the study and written informed consent was obtained from each patient. Between January 2006 and December 2015, 1753 adult kidney transplants were performed at Necker Hospital. Among these, we identified four transplant recipients with MELAS/MIDD syndrome (baseline characteristics are summarized in Table 1). After transplantation, patients were monitored monthly during the first year, then every three months. Each patient received an immunosuppressive regimen based on calcineurin inhibitors, steroids, and purine inhibitors. In all patients we stopped the calcineurin inhibitors for one week (wash out period) before switching to mTOR inhibitors. After the switch, patients were followed weekly for a month, bi-weekly for a month, monthly for the next 12 months, and then every 3 months. At all time-points, patients had physical examinations, performance status scoring using the Karnofsky $^{14,15}$ (on a scale from 0 to 100 , with lower numbers indicating greater disability) and the Eastern Cooperative Oncology Group ${ }^{14,15}$ (ECOG) indexes (a scale of 0 to 5 , with 0 indicating no symptoms and higher scores indicating increasing symptoms), and biological samplings. Sera from the four patients were collected every year after transplantation, before the switch to the mTOR inhibitors and then for all visits after switch.

\section{Generation of primary dermal fibroblast cultures}

Punch skin biopsies were collected from patients and healthy control individuals using standard methods. To generate dermal fibroblast cultures, biopsies were minced and incubated at room temperature in $0.05 \%$ trypsin-EDTA (ThermoFisher 25300054) solution for 30 min with gentle shaking. Cells were collected by centrifuging at $700 \mathrm{~g}$ for $10 \mathrm{~min}$, re-suspended in cell culture media containing $25 \%$ FBS, and plated onto 24 well plates to establish lines. Fibroblast cultures 
were grown and maintained in 1X MEM (Corning 10-010-CV) supplemented with 25\% FBS and penicillin/streptomycin (Corning 30-001-CI) to a final concentration of $100 \mathrm{IU}$ penicillin and 500 $\mu \mathrm{g} / \mathrm{mL}$ streptomycin.

\section{Pediatric Cell Lines}

Pediatric mitochondrial disease cell lines were obtained from the Coriell Institute Cell Repository. Pediatric lines are as follows: LS - Leigh Syndrome, ACH - ataxia and cerebellar hypoplasia, complex I, KSS - Kearns-Sayre Syndrome. The Coriell cell line ID's are GM01503, GM03672, GM13411, GM24529, GM06225, respectively. See Supplementary Table 2 for addition cell line details. All cell line characterization, including patient data and relevant cell line publications, can be found on the Coriell repository website by searching the cell line ID and opening the catalog data for the fibroblast cell line matching the identification (https://www.coriell.org/0/Sections/Search/).

\section{Analysis of mitochondrial membrane potential and morphology}

Cells at similar population doubling (PD 10 +/- 2) were plated 1:4 from confluent cultures onto coverglass chamberslides and allowed to grow until $\sim 80 \%$ confluent. Media was replaced and supplemented with $100 \mathrm{ng} / \mathrm{mL}$ rapamycin (Fisher BP29631) in DMSO (Fisher BP321-1), or equal volume DMSO, for 48 hours. Cells were stained for $15 \mathrm{~min}$ in media with $100 \mathrm{nM}$ tetramethylrhodamine ethyl ester (TMRE, Fisher BDB564696) and $5 \mu \mathrm{g} / \mathrm{mL}$ Hoechst 33342 (Biotium 89139-126) and imaged on a Leica SP5 confocal microscope. Samples were treated and imaged in one session using identical imaging parameters. Flow cytometry analysis was performed by staining cells with only TMRE or 10-N-nonyl acridine orange (10-NAO), dissociating with trypsin-EDTA containing dye for $10 \mathrm{~min}$ at room temperature, collecting cells by centrifugation, resuspension in cold PBS, and analysis on a BD Canto II flow cytometer using 
488nm excitation with 585/42 BP and 530/30 BP filters for TMRE and 10-NAO, respectively. A single gate was set to cells using forward and side scatter and all settings unchanged throughout data collection. 10,000 or more events were collected for every sample.

\section{Analysis of S6 phosphorylation by western blotting}

Cells at similar population doubling (PD 10 +/- 2) were plated 1:4 from confluent cultures and allowed to grow until $\sim 80 \%$ confluent. Media was replaced with media containing $100 \mathrm{ng} / \mathrm{mL}$ rapamycin in DMSO, 200ng/mL cyclosporin A (R\&D Systems 1101/100) in DMSO, or equal volume DMSO for 48 hours. Cultures were rinsed with 1XPBS, treated for 10 min with $0.05 \%$ Trypsin, collected by centrifugation at $4^{\circ} \mathrm{C}$, and pellets were flash frozen on dry ice. Protein lysates were collected by directly adding 1X RIPA buffer (Pierce 89900) containing protease and phosphatase inhibitors (Pierce PI78441) to cell pellets, sonicating in 10 one-second bursts, on ice, with an XL-2000 QSonica at maximum output, and centrifuging to remove cell debris. Protein concentration was determined by BCA assay (Pierce PI23228), equal protein run on 4$12 \%$ bis-tris 26 well NuPage midigels (Fisher WG1403), and transferred to nitrocellulose blots (Fisher IB23001). Blots were blocked in LICOR Odyssey blocking buffer (LICOR 427-40100), probed with primary antibodies anti-pS6, anti-S6, and anti-GAPDH (Cell Signaling 4858P, 2217S, and 2118S, respectively) followed by secondary antibody IRDye800 donkey anti-rabbit (LICOR 925-32213) and imaged using LICOR Odyssey Clx scanning imager as previously described $^{16}$. Data quantified using NIH Image ${ }^{17}$.

\section{Replicative capacity studies}

Excluding Supplementary Fig. 3 all cell studies presented were performed in 1X MEM (Corning 10-010-CV) supplemented with 25\% FBS and penicillin/streptomycin (Corning 30-001-CI) at a final concentration of $100 \mathrm{IU}$ Penicillin and $500 \mu \mathrm{g} / \mathrm{mL}$ Streptomycin. Fibroblast replicative 
capacity studies were performed using standard methods ${ }^{18}$. Briefly, to start the replicative capacity studies, low population doubling (PD) cells at $\sim 80 \%$ confluence were treated with 100 $\mathrm{ng} / \mathrm{mL}$ rapamycin in DMSO, 200ng/mL cyclosporin A in DMSO, or equal volume DMSO for 48 hours. Cells were split 1:4 and capacity measured by splitting cells 1:4 (adding two PD) each time they reached confluence. Media was changed 2-3 times per week with fresh drugs. All drugs were stored in frozen aliquots to prevent degradation associated with freeze-thaw cycles. The lifespans in Supplementary Fig. 3 were performed as above with the exception that the cell media used was EMEM with $1.5 \mathrm{~g} / \mathrm{L}$ sodium bicarbonate, non-essential amino acids, Lglutamine, and pyruvate (Corning 10-009-CV) supplemented with 25\% FBS and penicillin/streptomycin.

\section{SA- $\beta$-Gal, Actin Cytoskeleton Staining, and Nuclear Blebbing}

Senescence associated beta-galactosidase (SA $\beta$-Gal) staining was performed using Abcam kit ab65351 according to manufacture specifications. Images were collected using a Zeiss Axiovert 40CFL brightfield inverted microscope. Fraction SA- $\beta$-Gal positive cells were determined by counting total and SA- $\beta-$ Gal stained cells in 3-6 20X fields per treatment per cell line. Average values were grouped to compare control and MELAS/MIDD fibroblasts. Cytoskeletal staining was performed by growing cells on glass coverslips, fixing cells for $10 \mathrm{~min}$ in $3.7 \%$ paraformaldehyde in PBS, permeabilizing in 1\% Triton-X 100 (Fisher AC327371000) for 5 min with gentle shaking, blocked for 30 in 1\% BSA (Fisher BP9700100), and stained in 1X PBS containing 1\% BSA, 5 U/mL Phalloidin-CF594 (Biotium 89427-138), and Hoechst 33342 (5 $\mu \mathrm{g} / \mathrm{mL}$ ) for $30 \mathrm{~min}$ at RT. Cells were washed three times with $1 \mathrm{XPBS}$, mounted onto slides using Everbrite mounting media containing $1 \mu \mathrm{g} / \mathrm{mL}$ Hoechst 33342, and imaged on a Zeiss Axiovert 40CFL brightfield inverted microscope with a $20 \mathrm{X}$ objective and DAPI and Texas Red filter 
cubes. All images were collected in one session using the same exposure settings. Actin (red) and DNA (blue) channel signal intensities were measured using ImageJ. The ratio of red to blue channel intensity was calculated in 3-6 20X fields per treatment per cell line. Average values were grouped to compare control and MELAS/MIDD fibroblasts.

Nuclear blebbing was assessed using images collected from the Phalloidin/Hoechst stained slides. The fraction of 'blebbed' nuclei was calculated in 3-6 20X fields per treatment per cell line. Averages for each sample were combined to compare treated and untreated control and MELAS/MIDD fibroblast lines.

\section{A3243G genotyping by Sanger sequencing and restriction digestion}

Total DNA was extracted using the phenol/chloroform/isoamyl alcohol method following manufacturer protocol (Sigma 25666). A 183 base-pair amplicon containing the A3243 locus was amplified using forward 5rimer GCGCCTTCCCCCGTAAATGATATCATCTCAACTTAG-3' and reverse primer 5'-

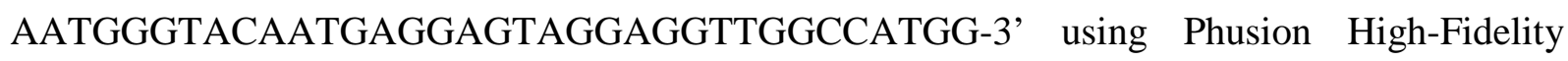
Polymerase (ThermoFisher F-530S) in a two-cycle PCR reaction. PCR products were submitted for Sanger sequencing by Macrogen (www.macrogenusa.com) and analyzed by restriction digestion using HaeIII according to manufacture specifications (NEB R0108S). Cleaved and uncleaved products were run on a 4-20\% TBE polyacrylamide gel (Fisher EC62255), stained with GelGreen DNA dye (Biotium 41007) and imaged using blue-fluorescent excitation wavelength (Supplementary Fig.9).

\section{Real-time quantitative PCR}

RNA was extracted from flash-frozen cell pellets using the Trizol method. cDNA was generated using the Superscript III First strand synthesis kit (ThermoFisher 18080051) according to 
manufacturer specifications. Expression of p16 (CDKN2A, p16INK4a) was assessed using Taqman probes (ThermoFisher) hs00923894_m1 (p16) and Hs99999907_m1 (B2M, beta-2microglobulin, a control gene), and the Taqman Gene Expression Master Mix (ThermoFisher 4369016), as directed, on an Applied Biosystems StepOne realtime PCR machine using the standard RT PCR protocol. Relative expression was determined using the delta-delta CT method.

\section{Serum metabolomics}

Serum samples were obtained at each visit (see above) and $50 \mathrm{uL}$ of serum was analyzed via LCQQQ-MS in MRM mode at the Northwest Metabolomics Research Center of the University of Washington. 128 metabolites were successfully identified in all samples.

\section{Glucose consumption}

Glucose consumption in Supplementary Fig. 6 was measured using a Bayer Contour glucose monitoring system with test strips (Fisher Scientific 23125600). Briefly, media was replaced 24 hours prior to splitting at confluence and glucose concentration was measured immediately before splitting cells. Consumption was calculated as final minus initial glucose mass in media.

\section{Statistical Analysis}

All data were presented as means +/- SEM. Comparisons between groups were performed using student t-tests, 2 -tails. $P<0.05$ was considered significant. Statistical comparisons of capacity curves were performed using the log-rank test as indicated.

For the metabolomics analysis, the $\log _{10}$ of the area under the curve for each metabolite was centered on the mean $\log _{10}$ and scaled on the standard deviation of each individual sample. Centered and scaled values were treated as repeated measures for each patient on either calcineurin inhibitors or mTOR inhibitors. 


\section{Acknowledgments}

This project has received funding from the European Research Council under the European Union's Horizon 2020 research and innovation program Grant 679254 (awarded to GC).

This work was supported by the Emmanuel Boussard Foundation (London, UK) and the Day Solvay Foundation (Paris, France), INSERM, Assistance Publique-Hôpitaux de Paris and University Paris Descartes.

This work was supported by NIH grants AG017242, GM104459, and CA180126 (awarded to YS). SCJ was supported by an American Federation for Aging Research (AFAR) Postdoctoral Fellowship and NIH grant F32 AG050444-02. BG was supported by NIH pre-doctoral training grant 6T32AG023475-13.

AB was supported by NIH training grant T32AG000057.

\section{COMPETING FINANCIAL INTERESTS}

The authors declare no competing financial interests. 


\section{References}

1. DiMauro S, Schon EA. Mitochondrial respiratory-chain diseases. N Engl J Med 2003;348:2656-68.

2. Schon EA, DiMauro S, Hirano M. Human mitochondrial DNA: roles of inherited and somatic mutations. Nat Rev Genet 2012;13:878-90.

3. Macmillan C, Lach B, Shoubridge EA. Variable distribution of mutant mitochondrial DNAs (tRNA(Leu[3243])) in tissues of symptomatic relatives with MELAS: the role of mitotic segregation. Neurology 1993;43:1586-90.

4. Koopman WJ, Willems PH, Smeitink JA. Monogenic mitochondrial disorders. N Engl J Med 2012;366:1132-41.

5. Laloi-Michelin M, Meas T, Ambonville C, et al. The clinical variability of maternally inherited diabetes and deafness is associated with the degree of heteroplasmy in blood leukocytes. The Journal of clinical endocrinology and metabolism 2009;94:3025-30.

6. Dimauro S, Mancuso M, Naini A. Mitochondrial encephalomyopathies: therapeutic approach. Ann N Y Acad Sci 2004;1011:232-45.

7. Schleit J, Johnson SC, Bennett CF, et al. Molecular mechanisms underlying genotypedependent responses to dietary restriction. Aging cell 2013;12:1050-61.

8. Johnson SC, Yanos ME, Kayser EB, et al. mTOR inhibition alleviates mitochondrial disease in a mouse model of Leigh syndrome. Science 2013;342:1524-8.

9. Johnson SC, Yanos ME, Bitto A, et al. Dose-dependent effects of mTOR inhibition on weight and mitochondrial disease in mice. Front Genet 2015;6:247.

10. Zheng X, Boyer L, Jin M, et al. Alleviation of neuronal energy deficiency by mTOR inhibition as a treatment for mitochondria-related neurodegeneration. Elife 2016;5. 
11. Kreis H, Oberbauer R, Campistol JM, et al. Long-term benefits with sirolimus-based therapy after early cyclosporine withdrawal. J Am Soc Nephrol 2004;15:809-17.

12. Canaud G, Bienaime F, Viau A, et al. AKT2 is essential to maintain podocyte viability and function during chronic kidney disease. Nat Med 2013;19:1288-96.

13. Wiley CD, Velarde MC, Lecot P, et al. Mitochondrial Dysfunction Induces Senescence with a Distinct Secretory Phenotype. Cell metabolism 2016;23:303-14.

14. Oken MM, Creech RH, Tormey DC, et al. Toxicity and response criteria of the Eastern Cooperative Oncology Group. American journal of clinical oncology 1982;5:649-55.

15. Velarde-Jurado E, Avila-Figueroa C. [Methodological considerations for evaluating quality of life]. Salud Publica Mex 2002;44:448-63.

16. Canaud G, Bienaime F, Tabarin F, et al. Inhibition of the mTORC pathway in the antiphospholipid syndrome. N Engl J Med 2014;371:303-12.

17. Schneider CA, Rasband WS, Eliceiri KW. NIH Image to ImageJ: 25 years of image analysis. Nature methods 2012;9:671-5.

18. Park SH, Kang HJ, Kim HS, et al. Higher DNA repair activity is related with longer replicative life span in mammalian embryonic fibroblast cells. Biogerontology 2011;12:565-79. 


\section{Figure Legends}

Figure 1: mTOR Activation and Mitochondrial Defects in Primary MELAS/MIDD Fibroblast Lines. A) Western blotting of lysates from primary MELAS/MIDD patient (MD mitochondrial disease) and control fibroblasts. Cells were treated for 48 hours with DMSO ('vehicle' solvent control), rapamycin (Rapa), or cyclosporin A (CsA) and lysates were probed for phosphorylated ribosomal protein S6, total rpS6, and GAPDH and quantification. B) Representative confocal microscopy images of primary fibroblast lines treated for 48 hours with DMSO or rapamycin and stained for mitochondrial membrane potential (tetramethylrhodamine ethyl ester, TMRE, red), mitochondrial mass (10-N-nonyl acridine orange, 10-NAO, green), and DNA (Hoechst 33342, blue). C) Representative flow cytometry analysis of primary fibroblasts treated for 48 hours with DMSO or rapamycin and stained for mitochondrial membrane potential (tetramethylrhodamine ethyl ester, TMRE, red). D) Schematic of m.3243A $>\mathrm{G}$ mutant mitochondrial DNA restriction analysis assay. E) Restriction analysis based assessment of m.3243A $>$ G heteroplasmy levels in cells treated for 48 hours with DMSO or rapamycin at two concentrations, 10 and $100 \mathrm{ng} / \mathrm{mL}$. F) Quantification of restriction analysis. 48 hours of rapamycin treatment has no impact on heteroplasmy levels, as anticipated. G) Western blotting of lysates from primary fibroblast derived from pediatric mitochondrial disease patients and control fibroblast obtained from the Coriell Institute Cell Repository and quantification. Pediatric lines are as follows: LS - Leigh Syndrome, ACH - ataxia and cerebellar hypoplasia, complex I, KSS - Kearns-Sayre Syndrome. $* \mathrm{P}<0.05, * * * \mathrm{P}<0.001$. Scale bar $\sim 10 \mu \mathrm{m}$.

Figure 2: mTOR Inhibition Attenuates Replicative Capacity Defects in Primary MELAS/MIDD Fibroblast Lines. A) Replicative capacity data from three controls and four MELAS/MIDD fibroblasts (MD - mitochondrial disease) treated with DMSO, rapamycin, or 
cyclosporin A (CsA). B) Relative change in replicative capacity in of CsA and rapamycin treated control and MELAS/MIDD primary fibroblast lines versus DMSO treatment. C) Representative senescence associated beta-galactosidase (SA $\beta$-Gal) staining of low population doubling DMSO and rapamycin treated MELAS/MIDD and control fibroblasts (PD: population doubling). D) Quantification of SA $\beta$-Gal staining (datapoints representive individual cell lines, 6 or more fields assessed for each line). E) Senescence associated factor $p 16^{I N K 4 a}$ (p16) displays increased expression in MD fibroblasts which is attenuated by 48 hours of rapamycin treatment. F) Representative images of normal nuclei and nuclei showing nuclear blebbing (arrows indicate nuclear invaginations or 'blebs'). Staning shows actin cytoskeleton (Phalloidin, red) and nuclei (Hoechst 33342, blue). Quantification of nuclear blebbing (datapoints representive individual cell lines, 6 or more fields assessed for each line). G) Staining for actin cytoskeletal organization (Phalloidin, red) and nuclei (Hoechst, blue). Quantification of nuclear cytoskeletal signal normalized to DNA content. Datapoints representive individual cell lines, 6 or more fields assessed for each line). H) Mitochondrial m.3243A $>$ G heteroplasmy analysis across the lifespan of MD line 2. Samples were collected every two population doublings and assessed together at the completion of the lifespan study. I) Quantification of K). Mitochondrial m.3243A>G heteroplasmy did not change in either DMSO or rapamycin treated cells throughout the duration of the lifespan studies. $* \mathrm{P}<0.05, * * \mathrm{P}<0.01 * * * \mathrm{P}<0.001$.

\section{Figure 3: Clinical and biological impact of mTOR inhibition in transplant recipients with}

MELAS/MIDD. A) Patient 1, B) Patient 2, C) Patient 3 and D) Patient 4. Pink parts of the graph are showing the period of exposure to calcineurin inhibitors (CNI) and in green the period of exposure to mTOR inhibitors (mTORi). The blue dots show body weight of the four patients over time, the black dots represent the serum albumin levels and the red dots the serum 
prealbumin levels. In patient 3 , the heart ejection fraction $(\mathrm{EF}, \%)$ before and after the switch to mTOR inhibitors is added. E) ECOG and F) Karnofsky scores at initiation of mTOR inhibitor treatment and at 18-20 month follow up. P-values calculated by paired t-test. G) $\log _{10}$ average difference of amino acid metabolites levels between samples on sirolimus/everolimus and on calcineurin inhibitors.

Supplementary Figure 1: Dose-dependent effects of rapamycin in fibroblasts. (A) Western blotting of phosphorylated and total S6 in control (WI38 line) fibroblasts treated with varying concentrations of rapamycin for 48 hours. IC50 of rapamycin for S6 phosphorylation was calculated to be $\sim 0.3 \mathrm{nM}$. (B) Relative glucose use in WI38 fibroblasts treated with varying doses of rapamycin for 48 hours. IC50 for rapamycin on glucose consumption is $\sim 2.6 \mathrm{nM}$.

Supplementary Figure 2: Additional western blot data. (A) Western blotting of phosphorylated and total PRAS40, a component of mTORC1 using samples shown in Figure 1. MELAS fibroblasts show a significant reduction in phosphorylated PRAS40 which is attenuated by 48 hours of rapamycin treatment. (B) Western blotting of phosphorylated and total PKC. There was a trend toward increased PKC phosphorylation (driven by 2 of the 4 lines) but this did not reach significance. (C) Western blotting of IGF1R processing and phospho-ERK (p42/44). IGF1R is produced as a pro-protein and processed by cleavage. MELAS fibroblasts have significantly lower levels of processed, compared to unprocessed, IGF1R, but this is not substantially altered by 48 hours of rapamycin treatment. Conversely, levels of phosphorylated ERK (p42/44 MAPK) are significantly increased by 48 hours of rapamycin treatment but are not different between the control and MELAS lines.

Supplementary Figure 3: Abnormal morphology and membrane potential of mitochondria in fibroblasts from patients with MELAS/MIDD. Representative images of abnormal 
mitochondria morphology and membrane potential in the fibroblasts from MELAS/MIDD patient, characterized by swelling, fragmentation, and the presence of depolarization as indicated by low TMRE (tetramethylrhodamine ethyl ester, red) staining relative to the less membrane potential sensitive dye 10-N-nonyl acridine orange (10-NAO). Nuclear DNA stained with the cell permeant dye Hoechst 33342 (blue). Low magnification in the left column and higher magnification in the right column. Arrows highlight overtly swollen mitochondria, as indicated by balloon or ball like appearance versus normal fibrillar organization. Scale bar $\sim 10 \mu \mathrm{m}$.

\section{Supplementary Figure 4: Mitochondrial and cytoskeletal defects in MELAS/MIDD}

fibroblasts. A) Mitochondria in MELAS/MIDD fibroblasts have collapsed and abnormal cristae and cells are characterized by high levels of cytoskeletal fibers. B) Mitochondria are visually more intact and organized in rapamycin treated MELAS/MIDD fibroblasts while cytoskeletal fibers are less distinct. Red arrows point to mitochondria with collapsed and abnormal cristae. Yellow arrows indicate mitochondria with healthy cristae morphology. Blue arrows point to regions of cytoskeletal fibers.

Supplementary Figure 5: Replicative lifespan in the presence of pyruvate. Addition of pyruvate attenuated the early growth retardation associated with rapamycin treatment but had no impact on the short lifespan of MELAS/MIDD cells or the increase in lifespan resulting from rapamycin treatment. A) Replicative lifespans of two control lines treated with DMSO or rapamycin in media supplemented with pyruvate. ${ }^{* * *} \mathrm{p}<0.0001$, paired $\mathrm{t}$-test DMSO versus rapamycin. B) Replicative lifespans of three MELAS/MIDD fibroblast lines treated with DMSO or rapamycin in media supplemented with pyruvate. ${ }^{*} \mathrm{p}=0.02$, paired t-test DMSO versus rapamycin. 
Supplementary Figure 6: p21 expression in fibroblasts from MELAS/MIDD. Western blot analysis of the senescence associated marker p21/Cip1 and quantification. Western blot performed at the start of the lifespan study, prior to onset of senescence. MELAS/MIDD fibroblast lines show substantially increased levels of p21/Cip1 compared to control fibroblast lines. $* \mathrm{P}<0.05$.

\section{Supplementary Figure 7: Metabolomic impact of mTOR inhibition in transplant recipients} with MELAS/MIDD. Heatmap of the $\log _{10}$ change of 128 serum metabolites before and after switch to mTOR inhibitors. Individual sample values per each metabolite were averaged across treatment per each patient. Data are represented as the difference between the average value on sirolimus/everolimus and the average value on calcineurin inhibitors period. Green: increase, red: decrease, white: unchanged. Black: $\mathrm{P}<0.05$, gray: $0.15<\mathrm{P}<0.05$.

Supplementary Figure 8: Glucose usage in primary fibroblast culture. Glucose useage in primary fibroblasts was robustly decreased by treatment with rapamycin but was not effected by treatment with cyclosporin A. Glucose consumption declined with replicative age in control and MELAS/MIDD cells treated with DMSO or cyclosporin; rapamycin treatment lowered consumption but also slowed the replicative age associated decline. Linear regression trendlines added when greater than 3 datapoints available. Glucose usage is reported in (ug glucose / hour / confluent $15 \mathrm{~cm}$ plate) (see Methods).

Supplementary Figure 9: Schematic of heteroplasmy analysis assay. A) Oligonucleotide primer sequences, amplicon sequence, and restriction site location. B) Schematic of restriction analysis band pattern. C) Restriction analysis of early passage patient and D) control fibroblast lines. E) Sanger sequencing chromatogram of the mutant site in the amplicon from patient 1. 

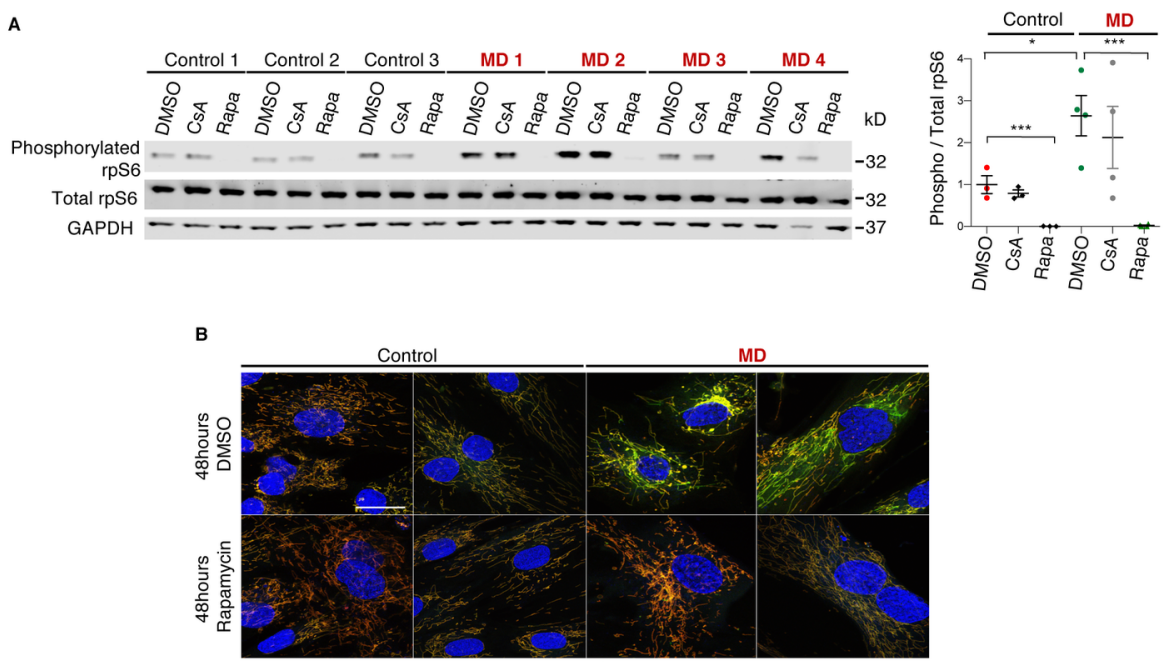

TMRE / 10-NAO / Hoechst

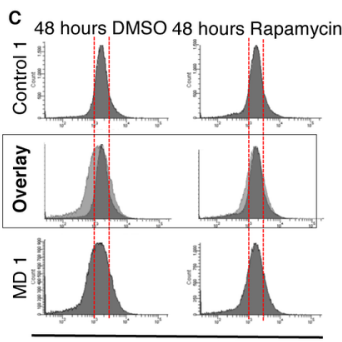

Stained with TMRE

(mitochondrial membrane potential)

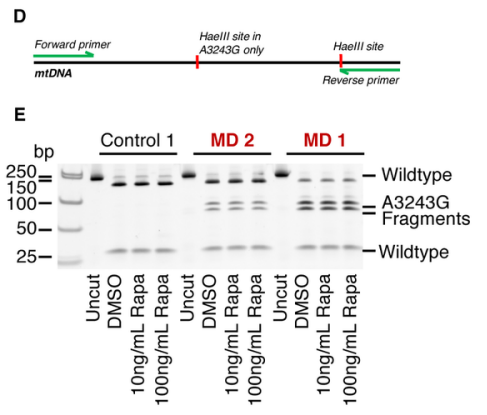

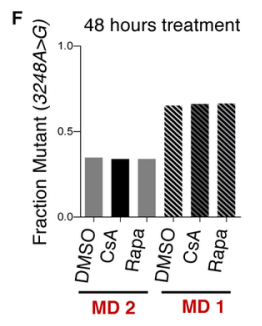

G

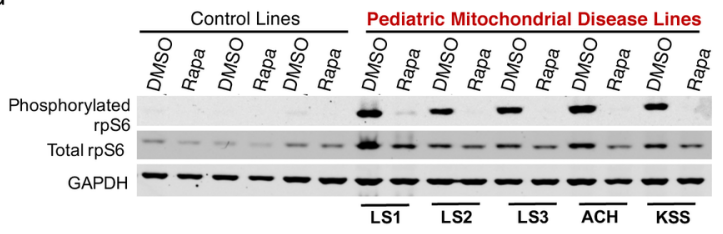

Control Pediatric

Lines Mito Disease

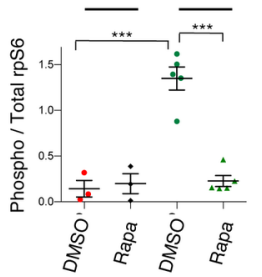


A

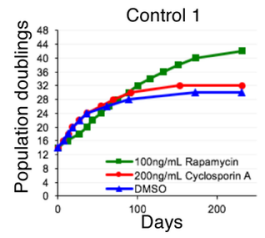

Control 2
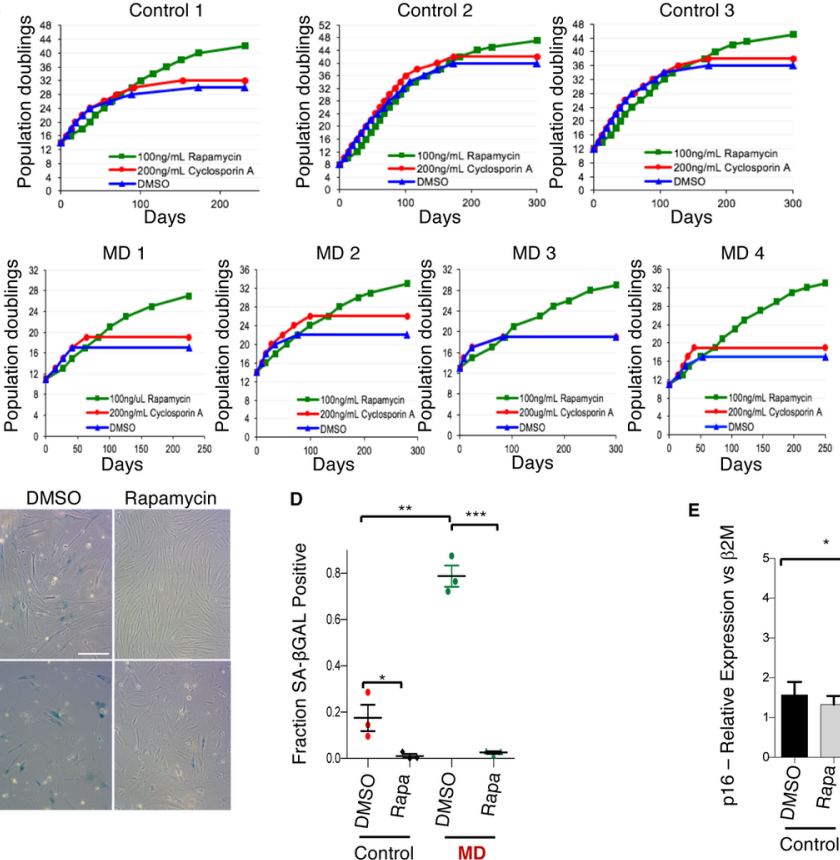

E

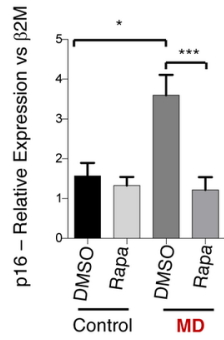

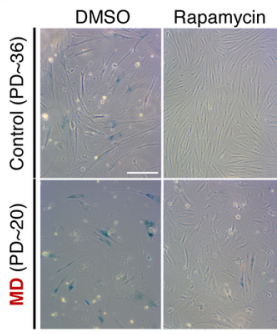

$\mathbf{F}$
Normal

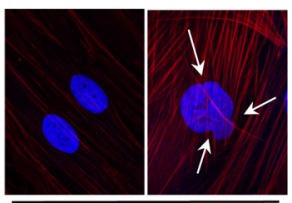

Phalloidin (Actin) / Hoechst (DNA)

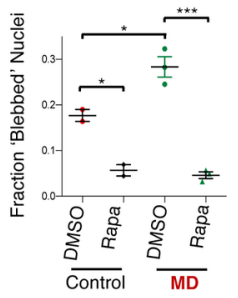

G

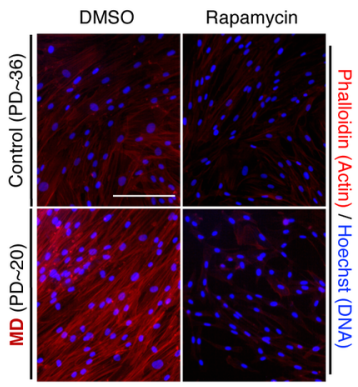

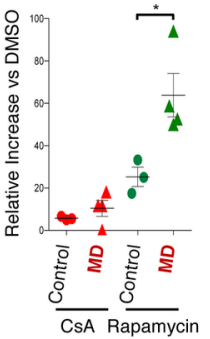




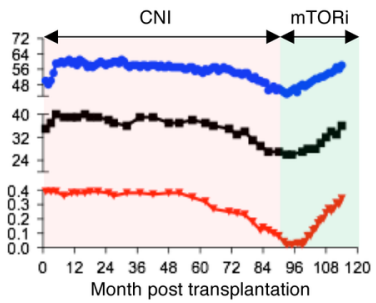

C

Patient 3

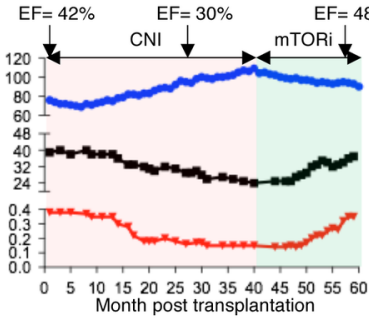

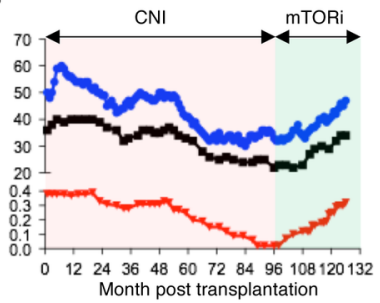

Patient 4

$\longrightarrow$ Body weight (kg- $\longrightarrow$ Serum albumin (g/L)두 Serum prealbumin (mg/L)

E

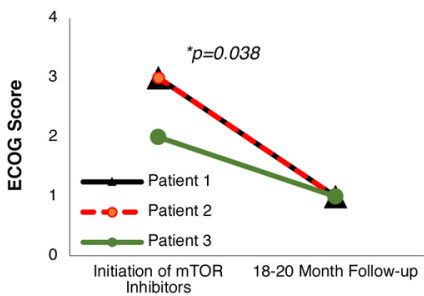

F

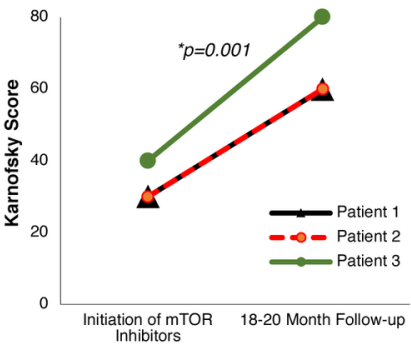

$\mathbf{G}$

3-Nitrotyrosine

Methylhistidine

Acetylcarnitine

Azelaic Acid

Myristic Acid

Margaric Acid

Linoleic Acid

Linolenic Acid

Malonic Acid

3HBA

Fumaric Acid

Succinate

Alpha Ketoglutarate

Aconitate

Glucose

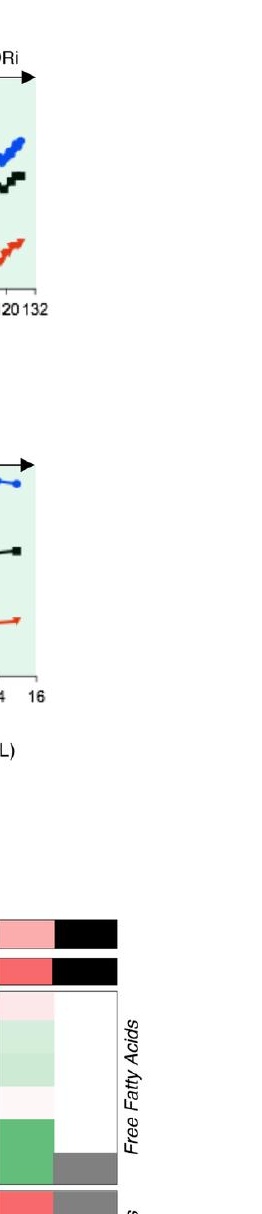

0
0
0
0
0
0
0
0
0

\section{政}

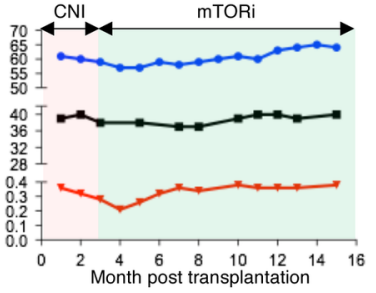


Table 1: Demographic and clinical characteristics of the patients

\begin{tabular}{|c|c|c|c|c|}
\hline Characteristics & Patient 1 & Patient 2 & Patient 3 & Patient 4 \\
\hline Age at diagnosis (years) & 34 & 42 & 33 & 44 \\
\hline Sex & Male & Female & Male & Male \\
\hline Sensorineural hearing loss & Yes & Yes & Yes & Yes \\
\hline Diabetes & Yes & Yes & Yes & Yes \\
\hline Heart dysfunction & No & No & Yes & No \\
\hline Muscle weakness & Yes & Yes & Yes & Yes \\
\hline Peripheral neuropathy & Yes & Yes & No & No \\
\hline Age at transplantation (years) & 44 & 52 & 51 & 59 \\
\hline $\begin{array}{l}\text { Immunosuppressive regimen } \\
\text { before switch }\end{array}$ & $\begin{array}{l}\text { Steroids/MMF/ } \\
\text { Tacrolimus }\end{array}$ & $\begin{array}{l}\text { Steroids/Aza/ } \\
\text { Cyclosporine }\end{array}$ & $\begin{array}{l}\text { Steroids/MMF/ } \\
\text { Tacrolimus }\end{array}$ & $\begin{array}{l}\text { Steroids/MMF/ } \\
\text { Tacrolimus }\end{array}$ \\
\hline $\begin{array}{l}\text { Switch to mTOR inhibitors after } \\
\text { transplantation (months) }\end{array}$ & 89 & 96 & 40 & 3 \\
\hline mTOR inhibitor after switch & Rapamycin & Everolimus & Rapamycin & Everolimus \\
\hline $\begin{array}{l}\text { Mean mTOR inhibitors trough } \\
\text { levels }(\mathrm{ng} / \mathrm{mL})\end{array}$ & $8 \pm 3$ & $7 \pm 3$ & $7 \pm 3$ & $8 \pm 2$ \\
\hline $\begin{array}{l}\text { Karnofsky (\%) / ECOG scores } \\
\text { before switch }\end{array}$ & $30 / 3$ & $30 / 3$ & $40 / 2$ & $90 / 1$ \\
\hline eGFR ( $\mathrm{mL} / \mathrm{min})$ before the switch & 72 & 78 & 56 & 74 \\
\hline eGFR (mL/min) after the switch & 74 & 79 & 60 & 74 \\
\hline HbA1c (\%) before the switch & 7.1 & 6.9 & 7.2 & 6.3 \\
\hline HbA1c (\%) after the switch & 6.9 & 7.0 & 7.1 & 6.5 \\
\hline $\begin{array}{l}\text { Cholesterol level before the switch } \\
(\mathrm{mg} / \mathrm{dl})\end{array}$ & 275 & 221 & 195 & 179 \\
\hline Cholesterol level after the switch & 332 & 296 & 251 & 227 \\
\hline $\begin{array}{l}\text { Triglyceride level before the switch } \\
(\mathrm{mg} / \mathrm{dl})\end{array}$ & 195 & 204 & 177 & 135 \\
\hline $\begin{array}{l}\text { Triglyceride level after the switch } \\
(\mathrm{mg} / \mathrm{dl})\end{array}$ & 231 & 267 & 243 & 195 \\
\hline
\end{tabular}


MMF: Mycofenolate mofetil; Aza: Azathioprine; ECOG: Eastern Cooperative Oncology Group; eGFR: estimated Glomerular Filtration Rate; Data are means \pm SD. 


\begin{tabular}{|c|c|c|c|c|c|c|c|c|}
\hline Cell Line ID & Description & Mutation(s) & Gender & $\begin{array}{c}\text { Age at } \\
\text { Sampling } \\
\text { (yrs) }\end{array}$ & Race & $\begin{array}{l}\text { Passage } \\
\text { Frozen }\end{array}$ & Publication & $\begin{array}{c}\mathrm{dbSNP} \\
\text { ID }\end{array}$ \\
\hline GM06225 & $\begin{array}{l}\text { Kearns-Sayre } \\
\text { Syndrome }\end{array}$ & - & Male & 10 & Caucasian & 5 & 15280047 & $\underline{14761}$ \\
\hline GM01503 & $\begin{array}{c}\text { Leigh } \\
\text { Syndrome }\end{array}$ & MTATP6 & Female & 3 & Caucasian & 5 & $\frac{2376596 ;}{\underline{7200213}}$ & 21779 \\
\hline GM03672 & $\begin{array}{c}\text { Leigh } \\
\text { Syndrome }\end{array}$ & MTATP6 & Female & 1 & Caucasian & 2 & $\underline{2376596}$ & $\underline{21068}$ \\
\hline GM13411 & $\begin{array}{l}\text { Leigh } \\
\text { Syndrome }\end{array}$ & $\begin{array}{c}\text { MTATP6; } \\
\text { 8993T>G mtDNA; } \\
\text { L156R }\end{array}$ & Male & 0.8 & Asian & 3 & $\frac{23665194 ;}{\underline{8042671}}$ & $\underline{11776}$ \\
\hline GM24529 & $\begin{array}{l}\text { Ataxia and } \\
\text { Cerebellar } \\
\text { Hypoplasia } \\
\text { Mitochondrial } \\
\text { Complex I } \\
\text { deficiency; } \\
\text { NUBPL }\end{array}$ & $\begin{array}{l}\text { NUBPL; T311C; } \\
\text { NADH:Ubiquinone } \\
\text { oxidoreductase } \\
\text { Fe/S protein; } \\
\text { Assembly of } \\
\text { Mitochondrial } \\
\text { complex I }\end{array}$ & Female & 14 & Caucasian & 2 & & \\
\hline
\end{tabular}

Table 2: Fibroblast cell lines obtained from the Coriell Institute Cell Repository 